\title{
El papel de los jóvenes en las ciudadanías alternas: un ejercicio de participación ambiental en contextos socioculturales diversos colombianos
}

\author{
The role of Young people in alternative citizenships: a \\ commitment to build environmental awareness in Colombian \\ sociocultural contexts ${ }^{2}$
}

\section{O papel dos jovens nas cidadanias alternas: um exercício de participação ambiental em contextos socioculturais diversos colombianos $^{3}$}

\author{
Catalina Quintero Ferrer \\ Magíster en Gestión Ambiental y Ecóloga \\ Fundación Universitaria del Área Andina \\ cquintero2@areandina.edu.co \\ Lorena Cardona Alarcón \\ Doctoranda en Literatura \\ Fundación Universitaria del Área Andina \\ lcardona46@areandina.edu.co \\ Romelia Negrete Doria \\ Doctora en Educación \\ Fundación Universitaria del Área Andina \\ rnegrete@areandina.edu.co
}

\footnotetext{
${ }_{1}$ El artículo se inscribe en el proyecto interdisciplinario de investigación de la Facultad de Educación: Infancia y juventudes: Comprensión de sentido y subjetividades desde territorios socioculturales diversos-fase I con código CVF 2020 -KO -B11, febrero-noviembre 2020.

${ }^{2}$ The article is registered on the interdisciplinary research project of the Faculty of Education: Childhood and youth: Understanding meaning and subjectivities from diverse sociocultural territoriesphase I with code CVF 2020 -KO -B11, February-November 2020.

$3 \mathrm{O}$ artigo se inscreve no projecto interdisciplinar de investigação da Faculdade de Educação: Infância e Juventudes: compreensão de sentido e subjetividades desde os territórios socioculturais diversos-fase I com o código CVF 2020 - KO -B11, fevereiro - novembro 2020.
} 


\title{
Resumen
}

Este artículo de investigación tuvo como objetivo la identificación de la forma cómo los jóvenes colombianos pertenecientes a contextos socioculturales diversos de las regiones Caribe y Andina. Los participantes de la investigación reconocen las principales problemáticas ambientales y se reconocen como actores en la toma de decisiones frente a la dimensión ambiental. Lo anterior es abordado desde el significado y las dinámicas de las ciudadanías alternas ejercidas entre los jóvenes desde la dimensión ambiental y la participación en toma de decisiones en sus territorios, por medio del activismo en espacios de formación o diálogo de saberes. La metodología es de corte mixto, con apoyo en grupos focales que cuentan con la participación de 32 jóvenes entre 14 y 25 años, estudiantes de básica media y educación superior. Los resultados se presentan a partir del análisis de las percepciones ambientales entre los jóvenes y el reconocimiento de las acciones de la población como agentes políticos en la toma de decisiones. De este modo, se visualiza el papel protagónico que asumen los jóvenes, con el interés en reconocer y apropiar los elementos biofísicos, sociales y políticos dentro de sus territorios, de manera que se conviertan en agentes transformadores a través de la acción y la participación.

Palabras claves: Dimensión ambiental, subjetividades, acciones, toma de decisiones.

\begin{abstract}
The objective of this research article was to identify how Colombian youths belong to diverse socio-cultural contexts in the Caribbean and Andean regions. The research participants recognize the major environmental issues and recognize themselves as actors in making decisions regarding the environmental dimension. It is approached from the meaning and dynamics of alternative citizenship exercised among youngsters from the environmental dimension, and participation in decision-making in their territories, through activism in training spaces or dialogue of knowledge. The methodology is mixed supported by focal groups that count with the participation of 32 youngsters between 15 and 25 years of age, high school, and higher education students. The results are presented based on the analysis of environmental perceptions among young people and the recognition of the population's actions as political agents in decisionmaking. In this way, the leading role assumed by young people is visualized, being interested in recognizing and appropriating the biophysical, social, and political elements within their territories so that they become transforming agents through action and participation.
\end{abstract}


Keywords: Environmental dimension, subjectivities, actions, decision making.

\section{Resumo}

O objetivo deste artigo de pesquisa foi identificar de que formas os jovens colombianos pertencem aos contextos culturais diversos das regiões Caribe e Andina. Os participantes da pesquisa reconhecem as principais problemáticas ambientais e se reconhecem como atores na toma de decisões frente a dimensão ambiental. O exposto é abordado desde o significado e das dinâmicas das cidadanias alternas exercidas entre os jovens desde a dimensão ambiental e a participação em toma de decisões em seus territórios, por meio do ativismo em espaços de formação ou diálogo de saberes. A metodologia é mista, com apoio de grupos focais que tem a participação de 32 jovens desde 14 até 25 anos, estudantes de básica meia e educação superior. Os achados se apresentam a partir das análises das percepções ambientais entre os jovens e o reconhecimento das ações da população como agentes políticos nas toma de decisões. Desta forma, visualiza-se o protagonismo assumido pelos jovens, com o interesse em reconhecer e apropriar os elementos biofísicos, sociais e políticos em seus territórios, para que se tornem agentes transformadores por meio da ação e da participação.

Palavras-chave: Dimensão ambiental, subjetividades, ações, toma de decisões.

\section{Introducción}

Los movimientos ambientalistas y los proyectos entorno a lo ambiental se han posicionado como un gran desafío y una necesidad a nivel mundial para mitigar el deterioro ecosistémico al que ha llevado el actual modelo de consumo, a las diferentes formas vivientes que habitamos el planeta. En este sentido, es imperativo reconocer la importancia de las percepciones frente a la dimensión ambiental en un grupo poblacional como los jóvenes, ya que son ellos quienes están movilizando estas iniciativas y generando unas nuevas miradas y otras alternativas ciudadanas desde esa perspectiva.

El mundo ve hoy como los ecosistemas se están fragmentando, poniendo en riesgo la supervivencia de múltiples especies y los bienes y servicios que se prestan a los humanos para vivir. Se identifican problemáticas como el cambio climático que se refleja en el aumento de al menos $1,0^{\circ} \mathrm{C}$ (WWF, 2020), generando mayor 
variabilidad climática, es decir, más temperaturas o precipitaciones. Sin embargo, se calcula que, con los modelos de vida actuales, los cambios planetarios podrán aumentar la vulnerabilidad. Por esto, las decisiones que se tomen por parte de los jóvenes pueden hacer la diferencia.

Entonces, ¿cómo los jóvenes colombianos pertenecientes a contextos socioculturales diversos de las regiones Caribe y Andina, se reconocen como actores en la toma de decisiones frente a la dimensión ambiental? Lo anterior, se analiza de acuerdo con las percepciones y espacios de participación establecidos, con el fin de promover y fomentarla, para generar cambios o alternativas en un país megadiverso como Colombia y comprendiendo que la dimensión ambiental involucra aspectos más allá de los elementos biofísicos y se relaciona con las interacciones sociales, culturales y económicas del territorio.

El punto de partida corresponde a Th. Marshall (1967), quien, después de analizar el caso inglés, presentó de forma general la noción de ciudadanía y sus elementos constitutivos.

El ensayo original, Ciudadanía y clase social, publicado en 1950, se constituye en el campo tradicional del concepto ciudadanía compuesto por derecho a tener derechos. El concepto alude, según
Marshall, a un estatus de plena pertenencia de los individuos a una sociedad y se confiere a quienes son miembros por plenos derechos en tres ámbitos: políticos, sociales y civiles. El primero a participar con el poder político; el segundo al ámbito del bienestar económico y la seguridad social; y, por último, el civil a los aspectos que abarca la libertad en todos sus ámbitos: pensamiento, individual, credo, propiedad, etc.

La ciudadanía -afirma- estaría compuesta por derechos cívicos y políticos, derechos de primera, segunda, tercera y cuarta generación, estos últimos referidos a la bioética. La crítica hecha a Marshall por otros autores se hizo notar frente a la concepción política y social de los derechos, por cuanto resultan insuficientes en el orden social vigente.

En suma, la ciudadanía como concepto en la democracia liberal se construye desde la línea del discurso de la modernidad, donde prevalecen concepciones sobre valores impuestos: libertad, igualdad, respeto activo, diálogo y solidaridad, los cuales han sido cuestionados por críticos ante la imposibilidad de alcanzarlos, ya que, la desigualdad que encierra la economía globalizada, conlleva a pensar la realidad de la ciudadanía de los participantes y la sociedad civil que, posiblemente, algunos conforman. 
La crisis del estado de bienestar permite, por un lado, recuperar el individualismo proclamado por el estado liberal de derecho, debido a que el ciudadano toma de nuevo su lugar, por encima de la ciudadanía colectiva, dando origen, por otro lado, a un diseño de un estado social de derecho, que asume el papel de participar antes que recibir, debido a que se conjugaba responsabilidad de ejercer la participación como un derecho inalienable.

Se trata un poco de que la ciudadanía redefina su poder de participación y los jóvenes como protagonistas activos dentro de la vida social y política - sobre la base de la noción de ciudadaníaconstituyan una categoría histórica, cuyo contenido ha estado en permanente transformación de una época a otra, pues ha dependido de condiciones socioculturales en las que se ha desarrollado. En este sentido, se ha reconstruido hacia un horizonte de participación y acción que, para el caso colombiano, definen retos interdisciplinarios desde las ciencias sociales.

La búsqueda de una participación ciudadana independiente, como afirman Roman y otros (2018), es un proceso de búsqueda alternativa y de participación que reconoce en los jóvenes, su capacidad de confrontación y construcción como agentes sociales $y$ generadores activos de nuevas posibilidades o ciudadanías alternativas.

Es así como los nuevos ciudadanos están vinculados al avance y desarrollo de los llamados movimientos sociales que, en los últimos años, han venido movilizando las bases conceptuales y de posiciones políticas, modificando propósitos con acciones que develan una nueva comprensión de lo que significa pertenecer a una comunidad sociopolítica y cultural, lo que permite una caracterización de nuevos elementos de la vida cotidiana y que habían sido relegados durante la modernidad, a esferas de la vida privada. Esta situación representa nuevas demandas de métodos de participación y el territorio no resulta ajeno a los jóvenes, sino que, por el contrario, es el escenario de acciones y toma de decisiones en su cuidado y preservación. Son estos nuevos elementos que inspiran o despiertan interés entre los jóvenes, constituyendo, de alguna manera, la ciudadanía del presente $y$ que interpela en su momento actual.

Ciudadanías que claman desde el presente por la participación

Las ciudadanías del presente se definen en relación con los problemas que tienen que enfrentar y resolver dentro del múltiple juego de poderes, y que exige pasar por mecanismos que 
se presentan como dicotomía básica entre los escenarios públicos y privados, tal como lo afirma Vives (2012):

Podemos decir que en el espacio privado predominan los intereses de los individuos, mientras que el espacio público se caracteriza por una justicia común para todos, que hace posible la convivencia entre la multiplicidad de interés privados a través de la mediación, que no responde a ningún interés específico y pretende satisfacer a los intereses generales. (pág. 85)

En efecto, los sujetos en una concepción liberal de la ciudadanía son partícipes primarios y siempre están a la espera de satisfacer sus necesidades, tema que demanda compromiso permanente, sin que se genere menoscabo, cuando desde una ciudadanía medioambiental aporta con sus deberes al servicio de la sociedad y/o de su entorno.

El cuidado del territorio como un bien público se convierte en el lugar para que los jóvenes, como ciudadanos, se manifiesten como individuos y sujetos de derechos y deberes. Por lo anterior, se considera que la participación de los jóvenes en el campo medioambiental puede incidir en la concepción que hoy se acepta de la sociedad. Al mismo tiempo, implicaría pensar un mundo sustentable a partir del relaciona- miento humano y la comprensión que existen en otras lógicas menos exacerbadas de consumo y estilos de vida, con poca valoración por el entorno que se agota, ante las posibilidades de garantizar la especie en el planeta. De esta forma, se puede entender la participación como ese conjunto de acciones que generan la toma de decisiones en aspectos que mejoran la vida en comunidad y también personal. Significa reconocer que las personas $y$, en particular para este estudio, los jóvenes se reconocen en el contexto de las relaciones en que viven y por lo tanto, es necesario contribuir al desarrollo de habilidades participativas, cada vez a más temprana edad, y no solo a partir de los parámetros establecidos para la llamada juventud, que requiere de estrategias más intencionadas y concretas.

En el caso colombiano, desde la Constitución Política de 1991 se ha buscado fomentar la participación ciudadana de todos los grupos sociales a través de mecanismos que buscan garantizar el derecho a la intervención de los ciudadanos en todas las actividades confiadas a las entidades públicas para la satisfacción de sus necesidades. Estos mecanismos establecidos en el artículo 103 se dan desde lo administrativo, lo judicial y lo político. 
La participación política, según Barnes, Case y colaboradores citados en Delfino, Gisela, Zubieta, Elena (2010)-, se define como "la acción realizada por ciudadanos con objetivos de influenciar las acciones o la composición del gobierno, que permiten exponer las preocupaciones frente al modelo actual".

Para el caso de la participación de los jóvenes, los mecanismos de carácter político son los más referenciados, siendo el voto el más reconocido, a pesar del alto porcentaje abstención. Según el DANE, en Colombia hay 8 millones de jóvenes entre los 18 y los 26 años -el 16\% de la población-, sin embargo, la Registraduría Nacional estima que en las últimas elecciones votaron solo 3 millones, lo que refleja una gran abstención de parte de los jóvenes.

A pesar de las tendencias abtencionistas, los jóvenes han encontrado espacios no formales de participación política como las marchas, movilizaciones, plantones, las asambleas y otros mecanismos. Esta participación política se ha promovido principalmente por las redes sociales, que se han convertido en puentes de comunicación e intercambio de realidades y preocupaciones comunes desde diferentes lugares y contextos.

La adecuación de la estrategia deberá basarse, en primer lugar, en los esfuerzos para incorporar la dimensión ambiental como sujeto político relevante, intrínseco a los planteamientos sobre el necesario nuevo modelo de evolución de las sociedades latinoamericanas, sin utilizar los términos "desarrollo" o "desarrollo sostenible", asociados a propuestas neoliberales. En la medida en que la aceleración del consumo de materiales siga llevando a la humanidad de catástrofe en catástrofe y el capital pueda reproducirse con independencia de los ecosistemas que destruya (y después de destruirlos pueda seguir reproduciéndose en otro lado), no será posible lograr la supervivencia de las futuras generaciones, ni siquiera de la presente. De ahí que se llame ciudadanías alternas a esas otras formas de solucionar problemáticas, resolver situaciones mediante acuerdos y consensos, sin tener que acudir a la ley o a marcos legales, lo cual indica que hay que pensar ¿cuáles son esas acciones que desde la juventud se pueden iniciar para dar cuenta del ser y hacer en un contexto?

Hoy se abre un inusitado espacio en la sociedad civil, un escenario donde confluyen la divergencia, la pluralidad y la diferencia para construir, expandir y reconocerse como jóvenes activos y protagonistas que, de alguna manera, desde la dimensión ambiental, contribuyen a reconfigurar el tejido social que 
imbrican desde lo local y regional. De allí la importancia que revisten contextos como el de la ciudad de Montería, Córdoba, y áreas aledañas a la región Caribe, donde se recogen percepciones de los jóvenes sobre la problemática ambiental local y de igual manera, sucede en territorios de la región Andina. Ese fue, precisamente, el marco de referencia donde la investigación encontró su pertinencia, en tanto la formación de ciudadanías alternas conduce a una dominación reiterada de derechos y deberes, en términos de lo que implica un proyecto societario actual.

Es claro que el debate hay que ponerlo en el tipo de estado que exige la sociedad, más eficiente y generador de democracia, comprometido con la formación de ciudadanía. Esto significa que aseguraría mayor participación y en este caso de juventudes creativas y con mayor ejercicio de poder, sentido deliberativo y condición de pertenencia a su territorio, reconociendo de este modo, otras posibilidades de ciudadanías alternas, más heterogéneas.

Ciudadanías alternas, abordaje de lo clásico a lo múltiple dimensional

La consideración que a partir de la modernidad se contrajo la idea de un dualismo ser humanonaturaleza es considerada desde la crítica a la modernidad como una nueva noción de ciudadanía alternativa, en tanto que busca la inclusión del otro que conduce a la redefinición sustantiva de aproximarse y comprender el entorno naturaleza y ciudadanía.

Retomando la idea sobre la versión liberal del estado como aparato maximizado de los derechos y libertades de los sujetos, donde la democracia funciona para la selección de gobernantes. Existen otras versiones $o$ vertientes de la democracia como la comunitarista -Robert Bellah, Charles Taylor, Michael Walzer-, pues piensa que las decisiones se construyen desde el campo de lo social. En este sentido, surge la ciudadanía ambiental como una suerte de academicismo que no aclara el horizonte de participación en escenarios como el rural. Este se atiene a la etimología del concepto de ciudadanía "polis" que, de alguna forma, amenaza los ambientes no urbanos. Desde esta perspectiva, otros abordajes han venido desarrollando una propuesta que busca superar las limitaciones del concepto para vincular, de manera más profunda, el tema ambiental. En ese contexto, tienen lugar apuestas por meta-ciudadanía ambiental o ecológica para construir un abanico de posibilidades, que no serán abordadas en el presente trabajo, dando paso en esas múltiples dimensiones, a las ciudadanías alternas como una propuesta en lo ambiental. 
En Colombia, la Ley 1549 de 2012 define la educación ambiental como un proceso dinámico y participativo, orientado a la formación de personas críticas y reflexivas, con capacidades para comprender las problemáticas ambientales de sus contextos locales, regionales y nacionales. Al igual que para participar de forma activa, en la construcción de apuestas integrales -técnicas, políticas, pedagógicas y otrasque apunten a la transformación de su realidad, en función del propósito de construcción de sociedades ambientalmente sustentables y socialmente justas. De acuerdo con lo anterior, la responsabilidad de actuar frente al medio ambiente se construye desde cada nivel social y etario, con una visión de futuro.

La dimensión ambiental se convierte, entonces, en un elemento integrador donde se interrelacionan los componentes sociales, económicos y biofísico, y dónde se articula con el componente político, al ser.

\section{ODS, jóvenes y ambiente}

Desde el año 1968, el hombre es consciente de que solo existe un planeta para vivir y por esta razón, durante los últimos 50 años, se han llevado a cabo espacios de actuación global para cuidar el planeta. Sin embargo, los avances en la protección del ambiente han sido insuficientes. En el año 2015, durante la reunión general de las Naciones Unidas, los países pertenecientes establecieron 17 objetivos que para el 2030 que permitirán tener un mayor bienestar planetario.

Los Objetivos de Desarrollo Sostenible (ODS) son la continuidad de los Objetivos del Milenio que promovieron la implementación de programas para el mejoramiento de la calidad de vida, pero al llegar el tiempo establecido para el cumplimiento, se hizo necesario también, su complemento. Se presentan entonces como el mayor reto de la humanidad, al integrar las diferentes dimensiones que afectan la sociedad, teniendo la dimensión ambiental gran importancia, especialmente en el 6, 11, 13, 14 y 15 , que se relacionan con energía, ciudades sostenibles, acción por el clima y protección de ecosistemas terrestres y marino, respectivamente.

Lograr la comprensión de los ODS en la cotidianidad y localidad permitirá que se busquen espacios de acción, corresponsabilidad y veeduría desde los diferentes grupos de la sociedad. En este contexto, los jóvenes serán en 2030 quienes tomen las decisiones que marcarán el rumbo del planeta, manteniendo un modelo extractivista, poco resiliente $y$ segregador, o generando las transiciones socioecológicas para la sostenibilidad. 
Dimensión ambiental: apuesta y retos en interdependencia sociopolítica

El concepto de educación ambiental invita a reconocer un ejercicio de reflexión acerca de cómo se concibe la dimensión ambiental dentro de la participación, la toma de decisiones y el reconocimiento territorial. Vega (2013) presenta este concepto de la dimensión ambiental como el conocimiento y reconocimiento de los elementos biológicos, físicos de los ecosistemas, junto con las relaciones de interdependencia de la sociedad. Es decir, el hombre y la naturaleza. Por esto, se ha convertido en un tema de agendas y decisiones políticas a nivel internacional para que las sociedades tengan en cuenta sus territorios.

Contextualizar la dimensión ambiental desde la complejidad del territorio colombiano, debe iniciar desde el reconocimiento de las diversidades ecosistémicas y sociales que lo llevan a ser el país más biodiverso, en relación con su área. Esto genera que características propias del territorio, sean factores determinantes para la toma de decisiones en los diferentes niveles de la organización social.

También es necesario incluir las realidades educativas y económicas que permiten promover o facilitar la relación o conocimiento del manejo adecuado de los bienes y servicios ecosistémicos (Andrade, 2018). Lo anterior, nos lleva al análisis de la dimensión ambiental desde la visión sistémica que incorpora las relaciones de interdependencia y el reconocimiento de los elementos biofísicos, económicos, sociales y políticos del territorio basados en la búsqueda del desarrollo sostenible. Para esto, la incorporación del reconocimiento del territorio es fundamental para la apropiación y toma de decisiones acordes con las necesidades.

La educación es, entonces, la base para generar procesos de autogestión y participación que permitan una posición crítica y responsable frente al manejo de los ecosistemas (Strieder Philippsen et al., 2017).

En ese orden de ideas, esta investigación presenta la percepción de los jóvenes acerca de su territorio desde la visión ambiental y cómo sus acciones impactan el ecosistema, en el marco de su papel dentro de las ciudadanías alternas como actores con interés político que se vinculan como nuevos agentes que buscan construir movimientos y nuevas identidades sociales para canalizar, de manera alternativa, acciones de cambio.

Han tomado fuerza las teorías que impulsan una democracia participativa, que entiende las ciudadanías como una expresión de identidad política desde los 
principios del pluralismo, donde se define la libertad y la igualdad para todos (Araujo, 2016). Desde esta perspectiva se propician espacios de intercambio de pensamientos sobre tópicos de interés entre los jóvenes y sus congéneres denominado "Jóvenes y ciudadanías alternas" para fortalecer una mirada por la dimensión ambiental, cuyo objetivo encuentra en las percepciones de los jóvenes sobre lo ambiental, una apuesta por promover diálogos abiertos alrededor de problemáticas diversas en sus contextos culturales y que emergen de preocupaciones por construir otras ciudadanías como posibilidades críticas participativas encaminadas a la configuración de sujetos políticos y agentes de soluciones. En este sentido, se toman algunas concepciones históricas sobre el concepto de ciudadanía.

\section{Materiales y métodos}

Este ejercicio investigativo se realizó en el marco de un enfoque mixto, donde a través de la recolección de datos cualitativos y cuantitativos, se obtuvieron resultados para tener una comprensión mayor del fenómeno investigado (Hernández Sampieri, 2018, pág. 546), que permitió comprender la realidad desde la perspectiva de los participantes en su ambiente natural y de acuerdo con su contexto.
Se desarrollaron tres grupos focales para la obtención de resultados con la asistencia de 32 jóvenes entre los 14 y los 25 años, pertenecientes a las regiones Andina y Caribe de Colombia. La selección de los participantes tuvo como criterio de exclusión, además de la edad, estar vinculado al programa de jóvenes de ambiente del Ministerio de Ambiente y Desarrollo Sostenible y la región de residencia. Para la participación se socializó la ley de protección de datos y para los menores de edad, se solicitó consentimiento de los adultos responsables.

Los grupos focales se desarrollaron en diferentes tiempos, teniendo en promedio la asistencia de 11 jóvenes. La convocatoria se realizó a través de redes, correos electrónicos y de manera telefónica. Durante los encuentros se realizaron preguntas semiestructuradas que sirvieron de guía para el desarrollo de la temática ambiental y que permitieron conocer las percepciones y opiniones.

Las preguntas orientadoras fueron: ¿cuáles consideras que son las principales problemáticas ambientales de tu territorio?; ia los jóvenes les interesan los temas ambientales?; ¿crees que los jóvenes toman decisiones, frente al medio ambiente?; y ¿de qué manera te gustaría participar en acciones a favor del medio ambiente? Adicionalmente, se 
aplicó un cuestionario a una muestra aleatoria de participantes, para corroborar la información obtenida en los grupos focales.

Para el análisis de la información se identificaron criterios y resultados comunes en los espacios de interacción, con el fin de identificar el papel de los jóvenes en las decisiones ambientales.

\section{Resultados}

Los jóvenes cada día constituyen un grupo con mayor influencia en diferentes ámbitos de la sociedad, al tener una organización estructurada por las nuevas realidades que presentan los medios de comunicación. El tema ambiental no ha quedado por fuera, siendo un interés manifestado por medio de participación en espacios relacionados.

Dentro de los resultados obtenidos en esta investigación, "los jóvenes" se reconocen como individuos con interés colectivos frente a sus realidades locales, pero con conocimiento de las globales. Sin embargo, son muy críticos frente a su papel a través de las formas de participación, porque consideran que son vistos como rebeldes, soñadores e ilusos y expresan que su opinión, no se tiene en cuenta al momento de tomar decisiones desde los espacios de construcción participativa como planes o programas a nivel local, regional o nacional.

Nicolas, 21 años, participante del segundo grupo focal manifiesta que "nos sentimos en la capacidad de expresarnos en un ámbito diferente, dado que muchas veces cuando nosotros queremos dar o expresar esa opinión en términos públicos o hacia los adultos no nos toman en serio, quizás por el mero hecho de que somos jóvenes, o porque tenemos ese estéreo tipo, por eso quizás hasta esta pregunta para mi es hasta ofensiva, porque para muchos es como simplemente llegar a generalizar que no pues a los jóvenes les interesa mucho más lo más media meramente por el mismo ámbito en el que estamos $y$ en el que nos encontramos" (sic).

Identifican la participación en las redes sociales digitales como el espacio de encuentro, debate y construcción de sus intereses. Esto en la dimensión ambiental se relaciona con la influencia que las redes han tenido frente a problemáticas como el cambio climático, donde a través de la convocatoria, se han realizado eventos globales tan importantes como los Friday for future - "Viernes por el futuro". Para este grupo etario, los nuevos espacios digitales son los lugares para validar sus expresiones y posiciones; además, a través de la interacción digital y mediada por redes sociales, se pueden llevar a cabo 
acciones reales de transformación, de acuerdo con las problemáticas que han sido identificadas desde los diferentes ámbitos.

A través del análisis de los grupos focales, se pudo identificar que los jóvenes de las regiones Andina y Caribe, encuentran la dimensión ambiental como una preocupación fundamental en su presente. Asimismo, reconocen como principales problemáticas: el manejo actual de los residuos sólidos, la contaminación de las fuentes hídricas y la deforestación, la falta de educación ambiental, la contaminación del aire y la biodiversidad (ver Figura 1).

Para los jóvenes, la participación en temas ambientales está directamente relacionada con el conocimiento del contexto, que muchas veces, se da fuera de los espacios educativos y se presenta como una debilidad. Además, consideran que el papel del estado es educar, pero también brindar y promover los escenarios de participación para la toma de decisiones, más allá de la participación informativa. Sin embargo, consideran que su participación es aún débil si se tiene en cuenta que las decisiones que se tomen hoy afectarán su vida adulta. En este sentido, lo expresa Diego, oriundo de Barranquilla, con edad entre los 18 y 25 años y participante en el tercer grupo focal: "Considero que los jóvenes participamos poco, dado que actuar frente a una problemática ambiental no lo puede hacer solo una persona y a la mayoría de los jóvenes encuentran poco interés en estos temas, aunque cabe resaltar que hay jóvenes que si le dan la atención que requiere" (sic).

Las nuevas maneras de comunicación y convocatoria se convierten, entonces, en la estrategia más reconocida y validada por ellos, como espacio de comunicación, discusión, construcción y cambio.

La investigación también permitió identificar que, en Colombia, la participación ambiental de los jóvenes ha sido promovida desde la Constitución Política y la Ley 99 de 1993, por medio de la creación de programas como "Jóvenes de ambiente". En estos se desarrollan acciones regionales para mitigar o resolver las problemáticas identificadas. Para el 2020, el Ministerio de Ambiente y Desarrollo Sostenible confirma que más de 1900 jóvenes se han vinculado desde los nodos regionales $\mathrm{o}$ institucionales (MADS, 2020). Igualmente, fueron identificados en la investigación, los consejos de juventudes, culturales y las convocatorias a los diferentes grupos etarios que se dan para la formulación de políticas públicas o instrumentos de planeación local. 
Figura 1. Principales problemáticas ambientales identificadas.

\section{Problemáticas ambientales identificadas}

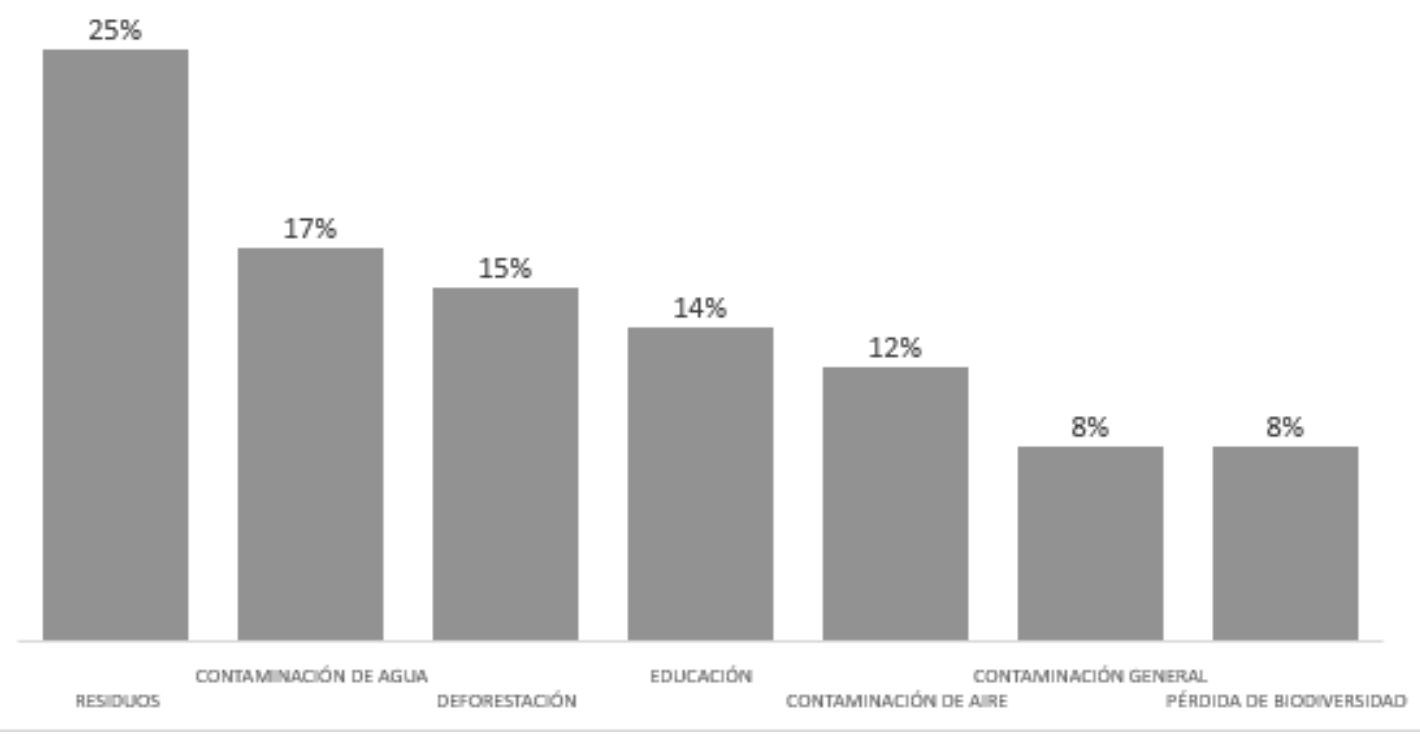

Frente a las estrategias de participación, se plantearon diferentes estrategias entre las que se encuentran las campañas, conversatorios académicos, colectivos o grupos ambientales, entre otros, siendo las campañas y grupos ambientales los predominantes con un 69.2\% de las respuestas (Figura 2).

\section{Discusión y conclusiones}

Las cuestiones relacionadas con la ciudadanía han venido cambiando. Por consiguiente, es importante señalar que el interés teórico por las cuestiones relacionadas con este tema se ha venido incrementando entre los jóvenes, para quienes sensibilizarse hacia políticas de participación resulta una aproximación cercana a pensar soluciones a la problemática sociopolítica de Colombia. Al respecto, Silva (2015) sostiene que, en la ciudadanía juvenil, se resalta el interés existente por temas como cultura y medio ambiente, proyectando a los jóvenes a futuro, siendo esta la diferencia con respecto a la ciudadanía adulta y, por ello, la juvenil es analizada desde diferentes perspectivas (pág. 279).

Se trata de una ciudadanía particular, cuyo ejercicio trasciende la apropiación de los mecanismos de participación para incursionar en tomas de decisiones y participación activa por asuntos culturales, sociales y ambientales. Al incursionar en otras posturas alternativas a la ya conocida definición clásica de ciudadanía, se acepta, en las 
prácticas actuales de Colombia, la postura de la ciudadanía civil como una suerte de convención política.

Se afianza un poco la idea de derecho sobre el ambiente, la cual está incorporada en el marco legal como derechos sociales y ambientales de cuarta generación, haciendo parte de expresiones con respaldo en gobiernos y políticas internacionales. De allí que la ciudadanía ambiental se sustente en ámbitos como la sostenibilidad ambiental que conducen a no pasar por alto los problemas que exigen soluciones urgentes. En esa línea y de primer orden, es necesario frenar la perspectiva consumista como un agente alterno a ser ciudadano, lo que conduce a potenciar una ciudadanía ambiental como un escenario de acción y toma de decisiones viables de ejercer en cualquier escenario o territorio, como otra forma de configurar sujetos políticos que dan origen a ontologías, en defensa de los contextos diversos en interdependencia con la dimensión ambiental.

Figura 2. Estrategias de participación

¿De qué manera te gustaria participar en acciones a favor del medio ambiente?

39 respuestas

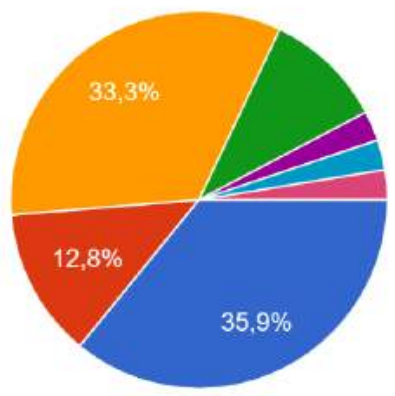

Participar en campañas

Conversatorios sobre medioambiente

Grupos ambientales

Gestor de medio ambiente

Más actividades como la que mencioné anteriormente, que no sea tanto de hablar sino de hacer y actuar ensegui...

Si tuviera la oportunidade gustaría participar activamente en los items an...

todas las anteriores

El rol de la participación en el futuro ambiental

Se ha encontrado que la participación de la sociedad en la toma de decisiones relacionadas con el ambiente es directa frente a los factores religiosos, la ubicación rural o urbana, las clases sociales y la proximidad o el conocimiento a problemáticas puntuales (He, 2020).

La participación efectiva de los jóvenes depende en gran medida de la educación ambiental brindada desde el aula, a través del conocimiento del territorio desde lo nacional, regional y local, donde la comprensión de la dimensión ambiental y su 
transversalidad sea tangible. Solo de esta manera y por medio de estrategias fomentadas $y$ articuladas desde lo institucional, tal como lo muestra el estudio realizado en San Salvador, Argentina, donde los estudiantes, en su mayoría, reconocen los bienes y servicios de los ecosistemas. Esto permite la apropiación del territorio y la toma de decisiones sobre él (Schaaf et al., 2018).

Por otra parte, para tener ciudadanos que estén dispuestos a cumplir con las normas, pagar impuestos $y$ tener un comportamiento adecuado con el ambiente, se requiere confianza institucional. Esta se forja por medio de la participación efectiva, más allá de las modas y tendencias y que debe ser promovida desde la sociedad misma (He, 2020). En este sentido se presenta también un reto a nivel nacional, porque los jóvenes no creen en la efectividad del estado para suplir las necesidades ambientales y sociales de un país que tiene dentro de sus estadísticas los asesinatos a líderes sociales y ambientales, la inequidad y la corrupción.

Como conclusiones derivadas del ejercicio de participación ambiental en contextos socioculturales diversos colombianos, se encontró que las problemáticas ambientales son reconocidas por los jóvenes desde los dos contextos. Sus percepcio- nes sobre lo ambiental se asocian al conocimiento del territorio desde los componentes sociales y biofísicos, entendiendo la relación directa entre los ecosistemas y la sociedad.

Las problemáticas, a pesar de ser regiones con características específicas, se centran en la educación ambiental, los residuos y la contaminación en general. Sin embargo, para los participantes de la región Caribe, la pérdida de biodiversidad genera mayor preocupación que en la Región Andina.

En relación con el territorio, el tipo de percepciones que tienen los jóvenes frente a lo ambiental hace un poco difícil que poblaciones dedicadas a la economía informal, abandonen la práctica de arrojar basura en sus entornos cercanos por donde se desplazan en su actividad de supervivencia. Esto genera otros problemas de insalubridad e inseguridad que hacen parte de la problemática sociopolítica en Colombia y que, en forma urgente, debe ser atendida por parte de organizaciones del estado y de organismos no gubernamentales como los movimientos en defensa del territorio, población y medio ambiente.

Frente a las estrategias de participación política y ciudadana se concluye que los grupos identifican las iniciativas no formales como los escenarios de 
acción y reconocimiento, donde, si bien no se toman decisiones, las ideas $\mathrm{y}$ posiciones pueden ser expresadas y socializadas para la construcción de ciudadanías alternas.

En ese orden de ideas y como afirma Ortiz (2010) "la exigencia en este campo demanda con urgencia un estructurado proceso de construcción de actores sociales, con capacidad de tomar decisiones en el escenario público el ejercicio de la ciudadanía, que integre los espacios y relaciones que devienen de lo político", ya que no son mediatizados por el estado. De acuerdo con lo anterior, Garretón (1999 citado por Ortiz, 2010) afirma que,

si la ciudadanía es el lugar del reconocimiento y la reivindicación de un sujeto de derecho frente a un determinado poder, $y$ este poder fue normalmente el Estado, hoy en día se generan campos o espacios en donde la gente hace el equivalente o la analogía con la ciudadanía. Quiere ejercer derechos, pero ese poder frente al que hay que conquistarlos ya no es necesariamente el Estado, o lo es solo parcialmente. (pág. 9)

Se requiere que desde los espacios de formación se promueva no solo la participación desde lo político, sino desde el cuidado común perteneciente a un territorio, sin que esto se convierta en un factor de riesgo. Asimismo, la identificación y formación de nuevos líderes sociales, políticos y ambientales conocedores, defensores y responsables desde la acción cotidiana, con visión colectiva y de sostenibilidad.

Se debe contemplar que la participación suceda desde una mirada política por parte de los jóvenes donde, en concordancia a Gligo (2020), la apropiación de la naturaleza se vea como un tema político que involucra las prácticas culturales y éstas, con el poder sobre el territorio. Por lo tanto, para actuar o generar procesos transformadores, se requiere un conocimiento y acción. En este sentido, un aporte fundamental de la investigación está en uno de los hallazgos principales en los contextos, el cual está relacionado con la estructuración de grupos medioambientalistas pertenecientes a comunidades escolares, otros en el ámbito universitario y local de algunas comunidades tal como lo arrojan los resultados encontrados en el grupo focal del presente estudio.

La investigación arrojó conclusiones que, sin lugar a duda, son fundamentales para continuar indagando y desarrollando el tema y generar nuevos debates de discusión. Con este propósito, invitamos a los lectores a trascender este texto y llevarlo a otros escenarios. Las conclusio- 
nes no son definitivas, pero pueden ser un referente y un camino para futuras investigaciones. En ese sentido, resulta claro que en el concepto de ciudadanías hoy, tiene fuerza la idea de otras ciudadanías alternas, donde juega un papel normativo independiente y que la promoción de la ciudadanía responsable, participativa, es un propósito de primer orden para toda política pública, teniendo en cuenta que el estado social de derecho tampoco ha llenado las expectativas de los ciudadanos.

Una de las razones para que los jóvenes se incorporen a organizaciones de corte civil y con preocupaciones ambientales se basa en poner en práctica el ejercicio de la ciudadanía participativa al ejercer ciertos derechos a la resistencia y la denuncia, al sentir que se atenta con el derecho a un medio ambiente sano.

Para abordar desde la participación de los jóvenes un impacto en las decisiones ambientales, se debe fortalecer la educación ambiental, de acuerdo con la normatividad, como un proceso, primero de reconocimiento, luego de reflexión crítica y participación activa en la toma de decisiones. Lo anterior fortalece los espacios de participación actuales y genera nuevas oportunidades a través de campañas o grupos desde los territorios; teniendo en cuenta como punto de partida, las preocupaciones y necesidades del ellos.

Es importante tener en cuenta que la ciudadanía alterna es el concepto que vincula a los individuos con otras formas de participación y de gestión en su comunidad y territorio, y se constituye en el nexo básico de participación, toma de decisiones. El reto hoy, en un contexto que presenta una fuerte tendencia, cada vez más creciente de participación de los jóvenes, es abrir espacios que den transformaciones inmediatas, a través de decisiones democráticas $y$ que den mayor cabida, a propuestas que convoquen unas ciudadanías alternas diversas desde todos los ámbitos, sin ninguna exclusión.

El reto está en la necesidad de reconocer la dimensión ambiental como parte fundamental de las generaciones actuales. Debido a esto, la disminución de los recursos naturales por la contaminación, por la pérdida de la biodiversidad o por la falta de conocimiento del territorio, se convierte a la vez en la pérdida de soberanía y de oportunidades sostenibles, en un país que es un punto de referencia en términos de biodiversidad, pero que hoy en día no ha podido suplir las necesidades básicas. Sin embargo, si no se toma acción hoy, el futuro está en riesgo, ya que, como lo afirma Gligo (2020, pág. 49), el discurso del medio ambiente se 
debe asumir como una tarea política y técnica que convoca a humanidad para un bienestar planetario.

\section{Referencias}

Andrade G. I., M. E. C. G. C. y C. T. (2018). Transiciones socioecológicas hacia la sostenibilidad. Gestión de la biodiversidad en los procesos de cambio en el territorio continental colombiano. Primera aproximación (M. E. C. G. C. y C. T. Andrade G. I., Ed.; IAvH).

Araujo. F. (2016). Los nuevos movimientos sociales y las ciudadanías emergentes. Estudios socio-jurídicos, 43-69. http://dx.doi.org/10.12804/revi stas.urosario.edu.co/sociojuridi cos/a.4025.

Gligo, N., Alonso, G., Barkin, D., Brailovsky, A., Brzovic, F., Carrizosa, J., ... y Villamil, J. J. (2020). La tragedia ambiental de América Latina y el Caribe. CEPAL.

Delfino, Gisela I, y Zubieta, Elena M. (2010). Participación política: concepto y modalidades. Anuario de Investigaciones, XVII, 211-220. [Fecha de Consulta: 17 de junio de 2021]. ISSN: 0329-5885. Disponible en: https://www.redalyc.org/articul o.oa?id=369139946011

Diario Oficial. (2012, Julio 5). Ley 1549 DE 2012. Diario Oficial.
Sistema Único de Información Normativa. Colombia, 6.

He, X. (2020). The role of social trust in public participation in environmental governance: Empirical evidence from households in China. Resources and Environmental Economics, 2(2).

https://doi.org/10.25082/REE.2 020.02 .005

Hernández Sampieri, R. (2018). Metodología de la investigación: las rutas cuantitativa, cualitativa y mixta. McGraw Hill.

Ministerio de Educación Nacional, Ministerio de Ambiente. (2002). Política Nacional de Educación Ambiental. Bogotá: Ministerio de Educación Nacional.

MADS. (2020, Julio 23). Respuesta derecha de petición SEP 8111-2465.

Ortiz, W. (2010). Ciudadanías alternativas. Una propuesta para el debate político actual. Revista Ratio juris, vol 5 No 11 (julio-diciembre 2010) pp. 69101.

https://doi.org/10.24142/raju.v 5n11a3

Mora, L. V. (2013). Dimensión ambiental, desarrollo sostenible y sostenibilidad ambiental del desarrollo. In Eleventh LACCEI Latin American and Caribbean Conference for Engineering and Technology 
"Innovation in Engineering, Technology and Education for Competitiveness and Prosperity" August (pp. 14-16).

Román, A., Plazas, D y otros (2018). Construcción de ciudadanías alternativas: apuestas y retos incluyentes en el escenario sociopolítico contemporáneo. Editado por Luisa Fernanda Muñoz Rodríguez. Universidad Manuela Beltrán, Bogotá.

Schaaf, A. A., Alcalde, S., Rivera, L., y Politi, N. (2018). Conocimiento de estudiantes primarios sobre el bosque y la biodiversidad de las Yungas Australes en la ciudad de San Salvador de Jujuy, Argentina. Ecología Austral, 28(3), 565-571. https://doi.org/10.25260/EA.18 .28.3.0.742
Silva, N., Cristancho, L. y otros (2015). Ciudadanía juvenil: Una breve revisión. Revista Universitas-Perspectivas en Psicología. Vol 11, No 2.

Strieder Philippsen, J., Soares Angeoletto, F. H., y Santana, R. G. (2017). El nivel de educación y los ingresos son importantes para la buena conciencia ambiental: un estudio de caso desde el sur de Brasil. Ecología Austral, $27(1)$ https://doi.org/10.25260/EA.17 .27.1.0.300

Vives, J. (2012) El ciudadano ecológico: Reflexiones sobre algunos contextos sociales y elementos cosmovisionales. Sociología y Tecnociencia. Revista digital de sociología del sistema tecnocientífico. 